\title{
Development Trends of Transmissions for Hybrid Electric Vehicles Using an Optimized Energy Management Strategy
}

\author{
Tianze Shi ${ }^{1,2} \cdot$ Fuquan Zhao ${ }^{1,2} \cdot \mathrm{Han} \mathrm{Hao}^{1,2} \cdot$ Zongwei Liu $^{1,2}$
}

Received: 19 August 2018 / Accepted: 3 November 2018 / Published online: 28 November 2018

(c) The Author(s) 2018

\begin{abstract}
Energy conservation and emissions reduction have become increasingly significant for automobiles due to the severity of the current energy situation. Hybrid electric vehicle (HEV) technology is one of the most promising solutions. This study investigated the total efficiency of a HEV powertrain. To improve the total efficiency, the engine should be regulated to work at its highest efficiency and drive the wheels directly as much as possible. To accomplish this, we developed an energy management strategy based on the direct drive area (DDA) of the engine's efficiency map. Several typical HEV models were built to compare the fuel consumption using DDA and rule-based strategies. Furthermore, the function of the HEV transmission system with DDA was considered. The transmission in a HEV should regulate the engine to work at its highest efficiency as much as possible, which is rather different than the regulation in an internal combustion engine vehicle. The functional change may lead to transmission systems with fewer gears but optimal gear ratios. If this trend is realized, the manufacturing cost of HEVs could be largely reduced.
\end{abstract}

Keywords HEV $\cdot$ Direct drive area $\cdot$ Energy management $\cdot$ Transmission cost reduction

\section{Abbreviations}

DDA

DP

CVT

EV

FC

HEV

ICE

ICEV

NEDC

P2 HEV

SOC

SP HEV

A

$C_{\mathrm{d}}$

$f$

\section{Zongwei Liu}

673262719@qq.com

1 Tsinghua Automotive Strategy Research Institute, Tsinghua University, Beijing 100084, China

2 State Key Laboratory of Automotive Safety and Energy, Tsinghua University, Beijing 100084, China

$\begin{array}{ll}I_{\mathrm{r}} & \text { Rotary inertia of wheel } \\ I_{\mathrm{e}} & \text { Rotary inertia of engine } \\ i_{\mathrm{T}} & \text { Transmission gear ratio } \\ i_{0} & \text { Gear ratio of final drive } \\ m & \text { Curb mass } \\ R & \text { Wheel radius } \\ u & \text { Vehicle running speed } \\ \eta & \text { Mechanical efficiency of power system }\end{array}$

\section{Introduction}

Transportation demands are increasing as modern cities continue to grow quickly [1-4]. This tendency leads to increased pollution, energy shortages, and other serious problems [5, 6]. Tailpipe emissions from vehicles must be controlled to enable sustainable development [7-11]. Efforts have been made in countries across the world to address the issues of large energy consumption in the transportation sector [12-14], including limiting transportation activity, restricting the purchase of automobiles, enacting strict emission regulations, improving vehicle fuel efficiency, and developing new energy sources for vehicles [15, 16]. Among the suggested solutions, the development of new energy sources 
is considered one of the most promising and practical methods [17-19]. Researchers and automotive manufactures have therefore committed a great effort to progress in this field.

A hybrid electric vehicle (HEV) is a complex system that integrates electronic, mechanical, chemical, and thermodynamic technologies. When a HEV is in motion, power and information are being transferred and transformed by various flows. The potential for HEVs to save energy and reduce emissions is determined by the means by which components are connected mechanically and the management of energy transfers between the internal combustion engine (ICE) and electric motor. The HEV onboard generator can charge the battery and recover energy by regenerative braking, allowing the battery to sustain an advantageous state of charge (SOC). A HEV that uses a smaller, more efficient ICE can achieve better fuel economy. The ICE can also operate within its most efficient operating area most of the time and can be switched off when necessary.

By combining the advantages of ICEs and electric vehicles (EVs), HEVs are quite promising for vehicle technology development in the short to mid term [20]. Lave and MacLean [21] compared a hybrid car to an ICE car and found that HEVs are not only effective for improving fuel economy and reducing emissions, but also, with gasoline price rising in the future, significantly reducing vehicle operating costs. Nordelof et al. [22] investigated the usefulness of different types of life-cycle assessments of HEVs and EVs to provide an overview of the environmental impacts. Hannan et al. [23] made a comprehensive review in the field of HEVs, concluding that existing technologies are capable of enabling good HEV performance, but that the reliability and "intelligence" of HEV systems are still somewhat inadequate. Cummings et al. [24] investigated the effect of sensitivity for sensing and prediction in vehicle fuel economy improvements. Morais et al. [25] presented distributed energy resource management using plug-in HEVs as a fuel-shifting demand response resource, which scheduled the EV's charging and discharging processes to avoiding network congestion. Chen et al. [26] investigated the influence of electrification on transportation and road systems. Lave and MacLean [21] made an environmental-economic evaluation between the HEV Toyota Prius and its ICE vehicle (ICEV) edition, the Corolla, pointing out that with fuel prices rising, the HEV would have significantly more market share in the future.

Different types of HEVs are classified by their degree of hybridization or their mechanical configuration. The main challenge for HEVs is splitting the power in an optimal way while delivering the desired performance under system constraints [27, 28]. Specifically, an energy management system is needed to select or combine the power sources for driving the vehicle [29-31]. Many different mechanical designs and energy management strategies have previously been presented by researchers using simulations and road tests [32-35]. Researchers have made great progress in regard to HEV general configurations, power electronic components [32, 33], and energy management strategies [34, 35]. The technology has developed rapidly and seen many breakthroughs. Morteza and Mehdi [36] developed a new energy management strategy for power splitting in HEVs using a multi-input fuzzy logic controller to further improve their fuel economy, tailpipe emissions, and performance in various driving cycles. Finesso et al. [37] applied an equivalent consumption minimization strategy to identify the optimal control strategy for a parallel HEV (P2 HEV). Li et al. [38] introduced a downshift strategy, and their hardware-inthe-loop simulation showed that this strategy can improve the energy conservation of HEV regenerative braking by $10 \%-32 \%$. Hannan et al. [39] studied a control system for a multi-energy source HEV. The control algorithm was developed to fulfill various driving conditions, and the simulation model [40] was built under the ECE-47 driving cycle. Their results showed that their multi-source control strategy could be efficient and conserve energy.

However, most research on the topic of HEVs is based on an architecture that uses traditional transmissions. Because the transmission system in a HEV should have functions different from those in an ICEV, its development direction should be different from that for ICEVs. In this context, this paper discusses the future direction of HEV transmission development.

\section{Modeling of Hybrid Electric Vehicles}

\subsection{Base Parameters}

In this study, several configurations of base ICEVs and P2 HEVs were modeled. The fuel consumption (FC) of these models was used as a reference baseline. To obtain the FC values, the energy consumption was calculated first with a vehicle dynamics formula. The formula includes rolling resistance, gradient resistance, aero resistance, acceleration resistance, and inertial resistance to model vehicle-specific running conditions and obtain vehicle-specific energy consumption. The vehicle dynamics formula is as follows:

$F=m g f+\frac{C_{\mathrm{d}} A u^{2}}{21.15}+m \frac{\mathrm{d} u}{\mathrm{~d} t}+\frac{I_{\mathrm{r}}+I_{\mathrm{e}} i_{\mathrm{T}}^{2} i_{0}^{2} \eta}{r^{2}} \frac{\mathrm{d} u}{\mathrm{~d} t}$,

where $F$ is the longitudinal force to drive the vehicle, $m$ is the curb weight, $g$ is the gravitational acceleration, $f$ is the rolling resistance coefficient, $C_{\mathrm{d}}$ is the drag coefficient, $A$ is the front face area, $\mathrm{u}$ is the running speed, $I_{\mathrm{r}}$ is the rotary inertia of the wheels, $I_{\mathrm{e}}$ is the rotary inertia of the engine, $r$ is the wheel radius, $i_{\mathrm{T}}$ is the transmission gear ratio, $i_{0}$ is the 
Table 1 Key parameters of the vehicle

\begin{tabular}{ll}
\hline Parameter & Value \\
\hline$m$ & $1386 \mathrm{~kg}$ \\
$R$ & $0.287 \mathrm{~m}$ \\
$C_{\mathrm{d}}$ & 0.30 \\
$A$ & $1.746 \mathrm{~m}^{2}$ \\
$f$ & 0.012 \\
$I_{\mathrm{r}}$ & $0.36 \mathrm{~kg} / \mathrm{m}^{2}$ \\
$I_{\mathrm{e}}$ & $0.46 \mathrm{~kg} / \mathrm{m}^{2}$ \\
$i_{0}$ & 4.5 \\
$\eta$ & 0.95 \\
$i_{1}$ & 2.804 \\
$i_{2}$ & 1.978 \\
$i_{3}$ & 1.531 \\
$i_{4}$ & 1.000 \\
$i_{5}$ & 0.705 \\
\hline
\end{tabular}

gear ratio of the final drive, and $\eta$ is the mechanical efficiency of the power system.

The New European Driving Cycle (NEDC) was applied to the calculation. The NEDC was established by the European Union and is applied in China. In the NEDC cycle, the vehicle is operated according to a given velocity curve. The total cycle lasts for $1200 \mathrm{~s}$ and has a top speed of $120 \mathrm{~km} / \mathrm{h}$. With vehicle velocity as input data, the force and power needed in a NEDC cycle for a typical car are obtained. The total energy consumption in a NEDC cycle is obtained by integration of the power data.

A typical mid-sized sedan was used in this study for the calculation. Key parameters of the vehicle are listed in Table 1 . The sedan was equipped with a five-speed transmission having the gear ratios shown in the table.

A typical ICE was used in the modeling, and its efficiency map is shown in Fig. 1. The engine has its highest efficiency of $40 \%$ at $2100 \mathrm{rpm}$ and $100 \mathrm{~N} \mathrm{~m}$. The FC rate of the engine at the optimal brake-specific fuel consumption was also obtained for use in the FC calculation.

\subsection{Rule-Based Energy Management}

The existing energy management strategies for HEVs can be mainly classified into rule-based and optimization-based management. Both types have been extensively studied. The researches have covered various aspects of these strategies, including the state of the art of energy management strategy, general formalization of the energy management problem, and characteristics and control effects of different strategies [32-39]. In an industrial application, rule-based strategies are more widespread. Although they cannot obtain an optimum solution, they are easy to implement because they have fixed rules. Thus, rule-based strategies have been successfully used

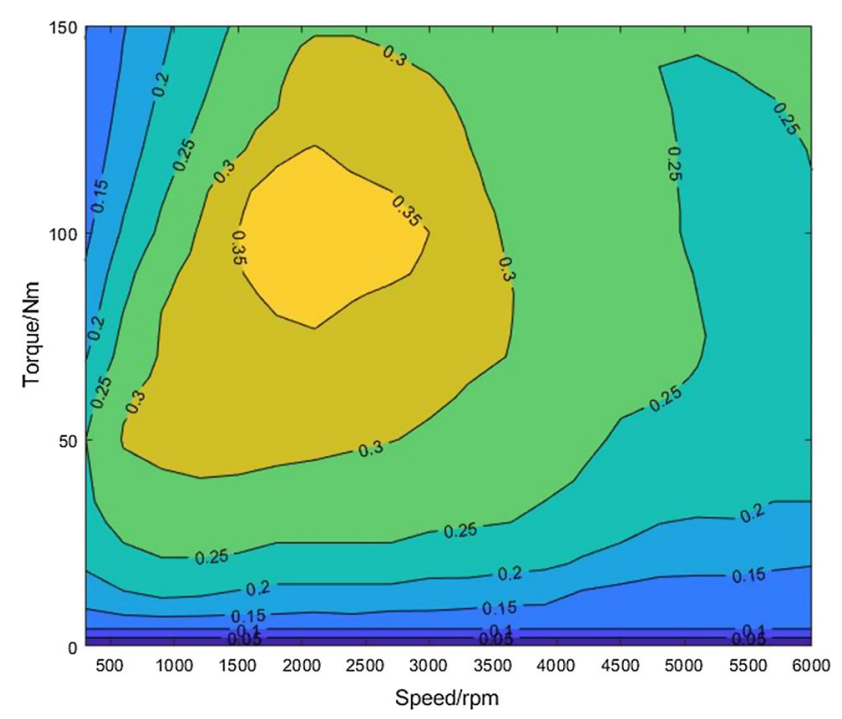

Fig. 1 Engine efficiency map

Table 2 FC values of baseline models

\begin{tabular}{ll}
\hline Model & FC $(\mathrm{L} / 100 \mathrm{~km})$ \\
\hline ICEV without trans. & 6.74 \\
ICEV with trans. & 6.48 \\
ICEV with CVT & 5.61 \\
P2 HEV without trans. & 4.75 \\
P2 HEV with trans. & 4.29 \\
Serial HEV & 4.13 \\
\hline
\end{tabular}

in commercial HEVs, and they provided the baseline for this study.

The logic flow of the rule-based strategy for P2 HEV s used in this study is as follows. First, the demand power $P$, demand torque $T_{\mathrm{r}}$, and acceleration $a_{\mathrm{cc}}$ are obtained as base demands. Second, the torques of best efficiency, motor working area, and maximum torque of the engine at a certain speed are determined. Third, the charging or discharging work mode is determined using the battery SOC. Finally, the demand engine and motor torque and battery charge or discharge state are obtained.

\subsection{Fuel Consumption Baselines}

With the necessary data and energy management strategy ready, the baseline ICEV and HEV models could be created. The NEDC condition was applied to calculate the FC, as implemented by the FC testing and evaluation provisions of the China National Standard.

The FC values of the baseline models are given in Table 2, including an ICEV without a transmission, ICEV with a five-speed transmission, ICEV with a continuously variable transmission (CVT), typical P2 HEV with rule-based energy 


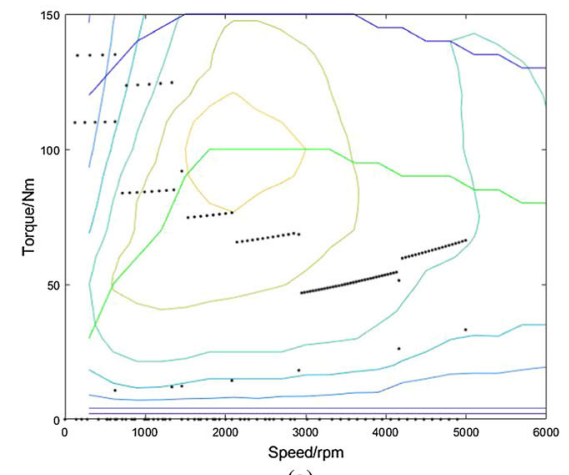

(a)

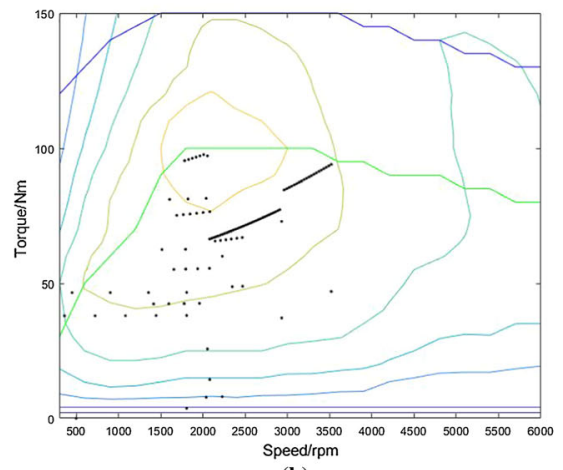

(b)

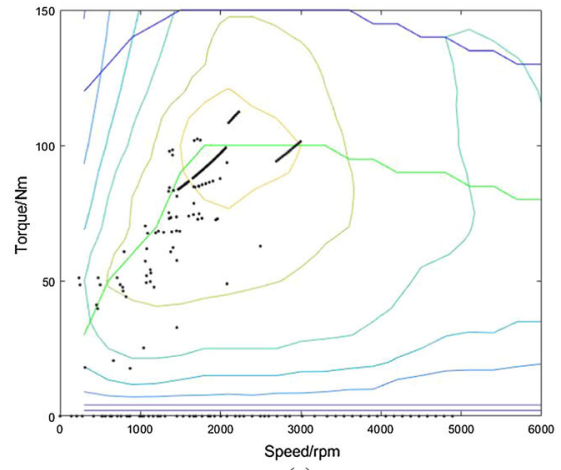

(c)

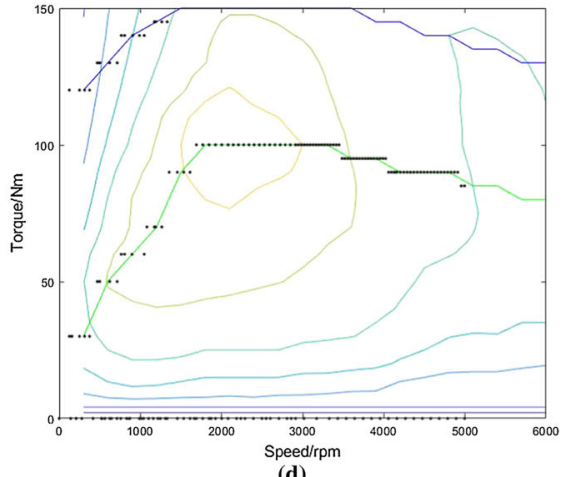

(d)

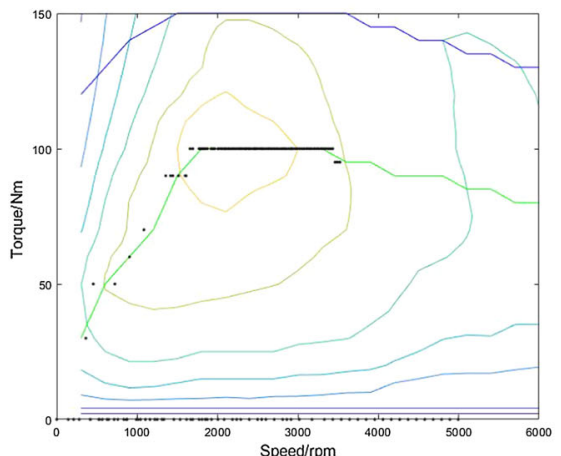

(e)

Fig. 2 Working points of baseline models. a ICEV without transmission. b ICEV with five-speed transmission. c ICEV with CVT. d P2 HEV without transmission. e P2 HEV with transmission

management, and serial HEV. The NEDC includes idle conditions for ICEVs, and the idle FC was set as $1.5 \mathrm{~L} / \mathrm{h}$, which is a typical value for an ICE in a sedan. For the HEVs, the engine shut down when the demand vehicle velocity was 0 and thus did not consume fuel when at a standstill.

The engine working points distribution for each model is shown in Fig. 2. The working points distribution can explain differences in FC values for the baseline models. For example, note that in Fig. 2a, some points are outside the engine full-load curve because this model has no transmission to regulate the speed and torque.

\section{Direct Drive Area (DDA) Energy Management}

\subsection{Improving Total Efficiency}

A HEV is driven by an ICE in combination with one or more electric motors. A battery package is connected to the motors as a secondary system to provide power to them. Reducing the FC of a HEV is equivalent to improving the efficiency of the HEV powertrain system, especially the working efficiency of the ICE. As the high-efficiency area of an engine efficiency map is rather small, engine speed/torque regulation is necessary to keep it working in this area.
However, regulating the engine working points to remain only at the best efficiency point is not the best solution. This regulation is usually done by motor torque adjustment. When the engine working point is regulated to the best efficiency point or line, the energy loss of motor and battery efficiency is inevitable. If the torque demand is less than the engine's best torque at a certain speed, the generator works and the energy is stored in the battery. Otherwise, if the torque demand is more than the engine's best torque, the motor works to provide the torque needed. Neither approach will cause energy loss in the motor drive or battery.

Thus, when the ICE drives the wheels directly, there is an area near the engine's best efficiency point in which the total ICE efficiency is higher than that obtained by the best efficiency regulation. We call this area the "direct drive area" (DDA), and it is determined by the efficiencies of the motor and battery charging/discharging cycle. If the motor and battery efficiencies are higher, the DDA will be larger; otherwise, it is smaller. The DDA of the engine used in this study is shown in Fig. 3. The outline of the DDA is obtained by multiplying the best efficiency value by the average motor efficiency and battery efficiency. Then, the DDA can be drawn as an engine efficiency map, as shown in Fig. 3.

In the DDA strategy, the engine will directly drive the wheels in the DDA, rather than being regulated to the best efficiency point. In this way, energy loss in the motor and bat- 


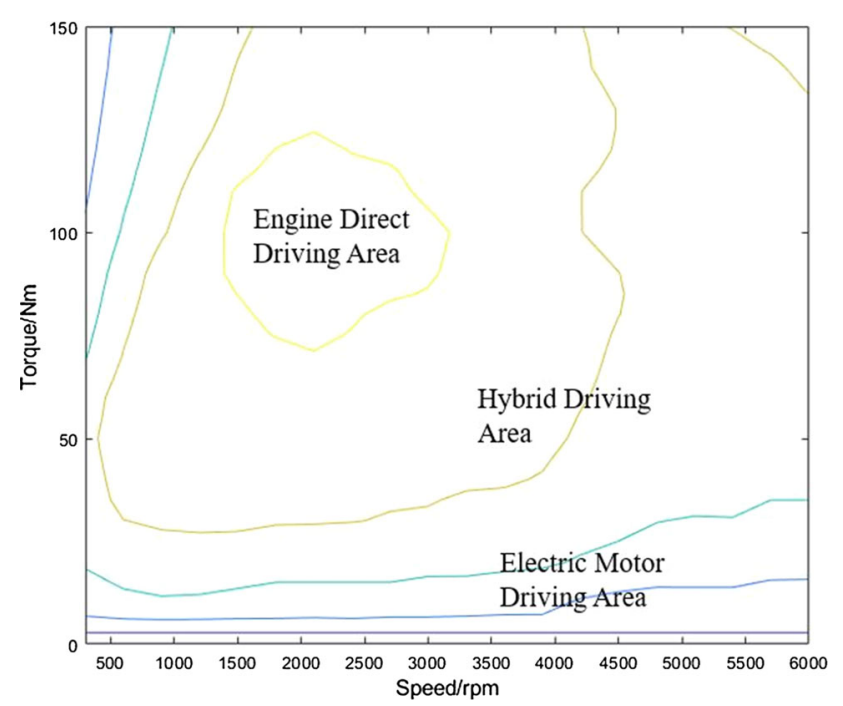

Fig. 3 Direct driving area

tery is avoided. Actually, similar results have been obtained using a dynamic programming (DP) method [27]. The working points regulated by the DP method are usually distributed near the DDA, and the results show good FC reduction. But the DP method can only be used when the whole working condition is already known, and the calculation speed is rather slow. Thus, it is not used in industrial applications. While the DDA method is a rule-based strategy, the DDA is determined in advance. During the driving cycle, the working point of the torque and speed is determined. If it is within the DDA, the engine directly drives the wheels; otherwise, the working point is regulated to the best efficiency point, with the motor assisting the power output.

\subsection{Application of DDA Method}

The DDA method can be realized in several ways. It can be applied in a typical P2 HEV or a serial-parallel (SP) HEV. The architecture of the SP HEV, which was developed by Honda Co., is shown in Fig. 4. When the engine working condition is in the high-efficiency area, the clutch is locked so that engine power directly drives the wheels. Otherwise, under low-speed conditions for instance, the engine efficiency is rather low and the clutch is released to drive the wheels only with the motor. The HEV system works as a serial HEV in this case.

To demonstrate that the SP HEV is a good application of the DDA, we built a model. Figure 5 shows the engine working point distribution of the SP HEV model without a transmission. The red square shows the engine DDA, outside of which the working points are regulated to the best efficiency point (as a serial HEV). Note that only few direct

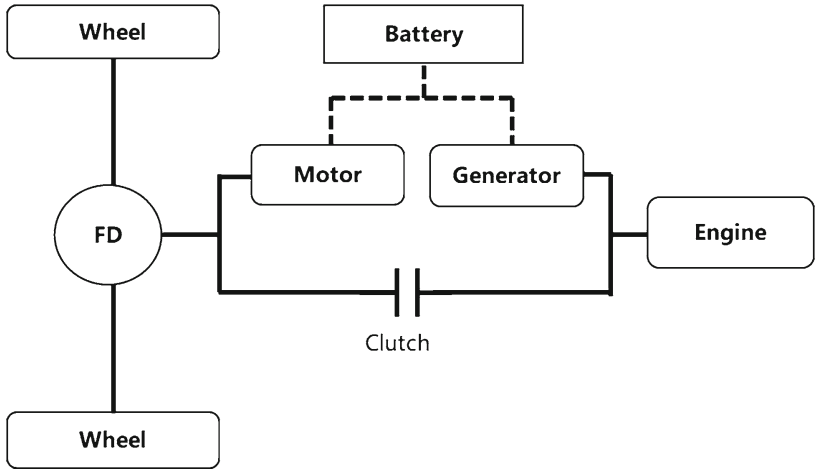

Fig. 4 Architecture of SP HEV

driving points are available in the NEDC because its driving load is mostly rather low. In the SP case, $\mathrm{FC}$ is $4.12 \mathrm{~L} / 100 \mathrm{~km}$, which compares with $4.13 \mathrm{~L} / 100 \mathrm{~km}$ in the serial HEV case. The slight improvement comes from the fewer direct driving points. When a high-load or high-speed working condition is considered, a better FC reduction may be achieved. Although the working point distribution in Fig. 5 is obtained by investigating a typical SP HEV model, it still provides the common features of an SP system.

DDA can also be applied to P2 HEVs. When a rule-based strategy is applied to $\mathrm{P} 2 \mathrm{HEVs}$, all engine working points are regulated to the best efficiency, including those in the DDA. In this study, the control strategy was changed using the rulebased strategy. For those points inside DDA, the ICE drives the wheel directly, as described above. The engine working points distribution when the DDA method is applied to a P2 HEV with a five-speed transmission is shown in Fig. 6b, comparing with the working points of $\mathrm{P} 2 \mathrm{HEV}$ without applying DDA in Fig. 6a. As can be seen, some of the points in Fig. 6b are away from the best efficiency line, when the ICE directly drives the wheels. Other points that are regulated to the best efficiency line in Fig. 6a are the points outside the DDA area, which can be found in Fig. 2b. In general, the FC of a P2 HEV controlled with the DDA method is $4.09 \mathrm{~L} / 100 \mathrm{~km}$, compared with the $4.29 \mathrm{~L} / 100 \mathrm{~km}$ of a rule-based P2 HEV and the $6.48 \mathrm{~L} / 100 \mathrm{~km}$ of a pure ICEV with a transmission. Note that the FC value is even lower than that for the SP HEV because the transmission regulates some working points into the DDA. If we add a transmission to the SP HEV to regulate the working points, the $\mathrm{FC}$ value will be lower than that for the P2 HEV.

\section{HEV Transmission Improvement Tendency}

\subsection{Functions of HEV Transmission}

Application of the DDA method changes the function of the transmission in a HEV. In an ICEV, the function of the transmission is to regulate the working point as close as possible 


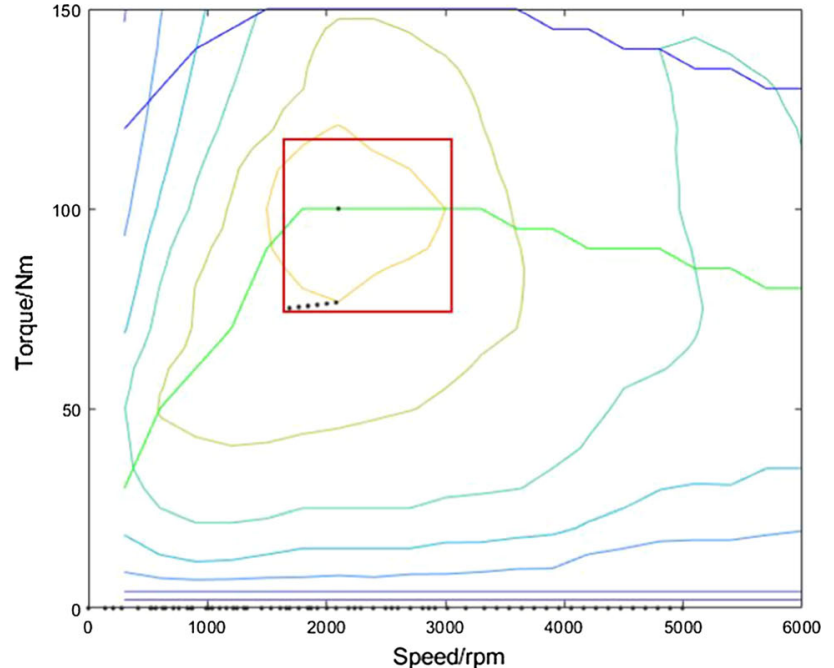

(a)

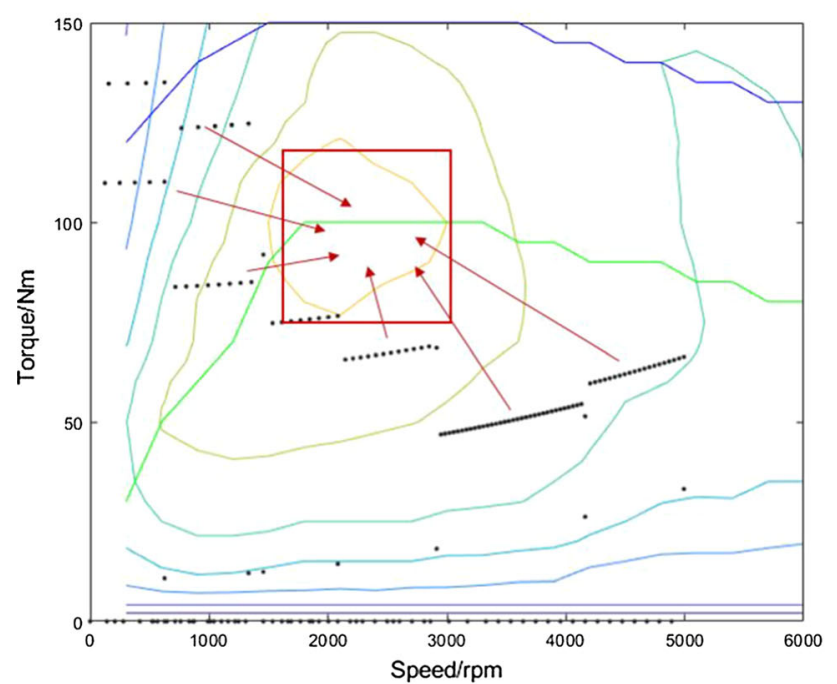

(b)

Fig. 5 Working point distribution of SP HEV. a Working points of SP $\mathrm{HEV}$ in DDA. b Working point regulation

to the best efficiency line of the engine at a given power. This means that more gears in a transmission result in better regulation. But more gears mean higher costs. Thus, most commercial transmissions have five or six gears. In theory, the CVT could achieve an infinite number of gears to realize the best efficiency point under any condition. However, the transmission efficiency of CVTs is rather low due to the application of a torque converter. Transmissions in ICEVs have already been thoroughly addressed along with the development of vehicles.

When an electric motor is introduced into vehicles, the function of the transmission changes, especially when applying the DDA method. In HEVs using the DDA method, the FC will be significantly reduced if there are more points in the DDA. The transmission could regulate engine work-

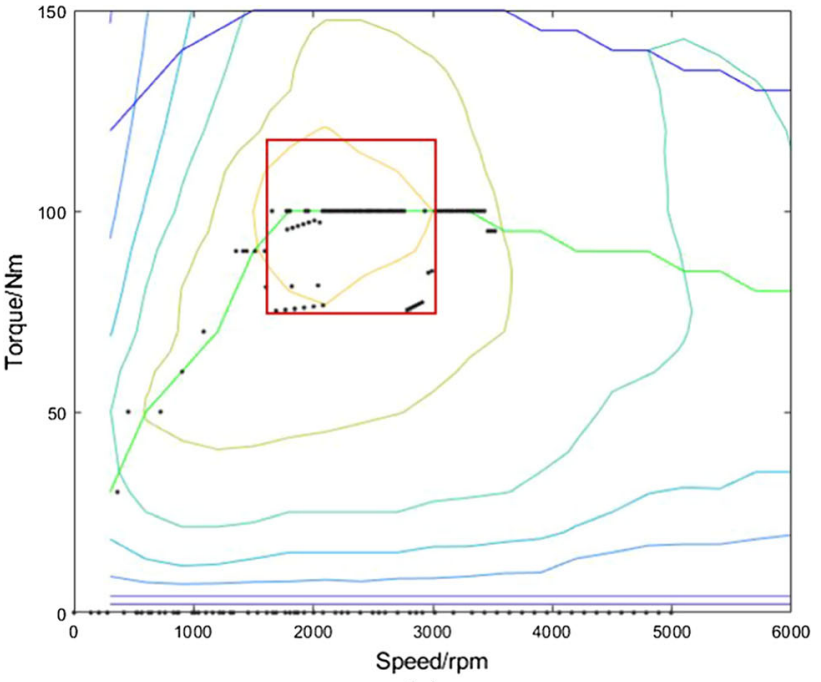

(a)

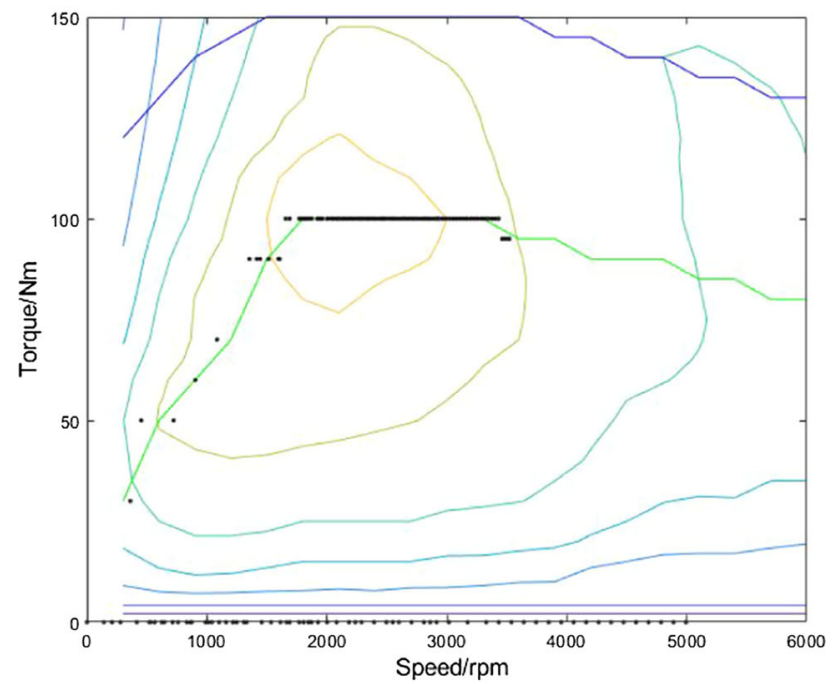

(b)

Fig. 6 Working point distribution of P2 HEV. a Working points of DDA method. b Working points of rule-based strategy

ing points to realize this. The function of transmission is to regulate a wide speed range (1500-3000 rpm, e.g.) to a rather narrow DDA area speed interval (500-600 rpm in this study). To illustrate the new function of a transmission in a HEV controlled with the DDA method, a typical five-speed transmission was added to the SP HEV model. The engine working point distribution is shown in Fig. 7, along with that of the baseline ICEV. It can be seen that more points are inside the DDA, where the wheels are directly driven. For other points, they are regulated to the engine's best efficiency point, as in a serial HEV. With more direct driving points, the FC value is $4.06 \mathrm{~L} / 100 \mathrm{~km}$, compared with $4.12 \mathrm{~L} / 100 \mathrm{~km}$ in a SP HEV without speed regulation.

As the engine working points regulated by the transmission follow a hyperbola, different operating profiles are revealed at higher and lower speeds. When regulating the 


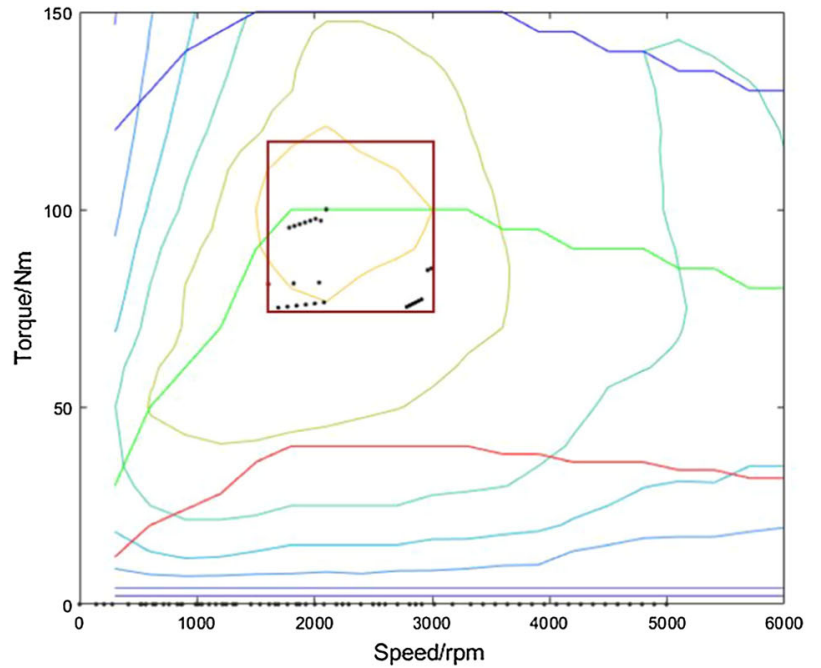

(a)

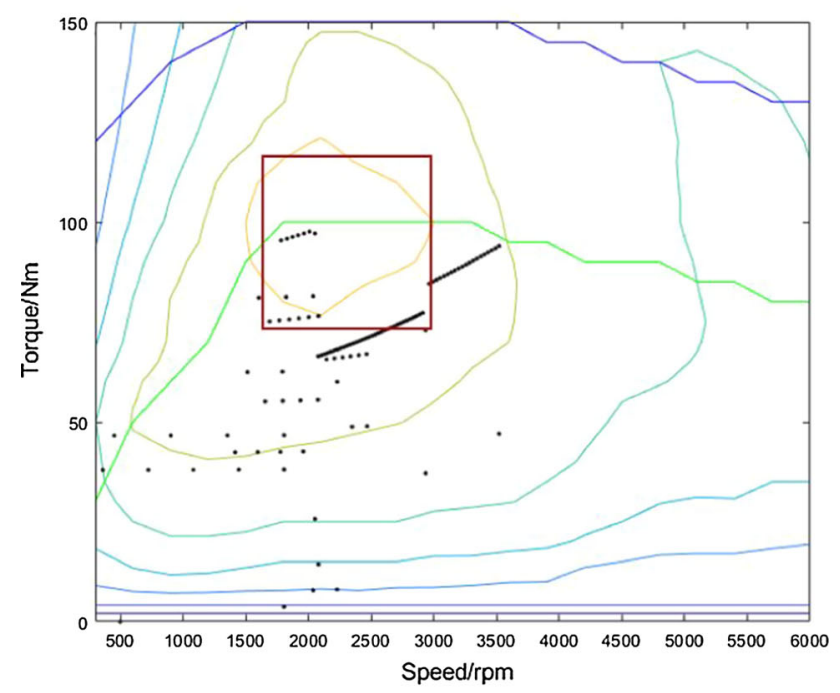

(b)

Fig. 7 Working point distribution of SP HEV with five-speed transmission. a SP HEV with transmission. b Baseline ICEV with transmission

higher-speed working points to lower speed, the torque will increase correspondingly to ensure the same power output. Because most high-speed conditions are associated with lower torque demands, points in this area could be regulated into the DDA with a high probability of better efficiency.

However, when regulating a lower-speed point to high speed, the torque decreases. If the original torque is low, then the regulated torque decreases and might fall out of DDA. In this case, the hybrid mode will be engaged. If the torque is too low, the electric mode becomes active and the vehicle is driven only by the motor. Otherwise, the hybrid mode is active, and the driving point is forced to the best torque/speed ratio that drives the wheels while charging the battery. Meanwhile, the battery SOC is also considered to decide which mode to select. In either case, the objective is to make the total efficiency higher than the original (unregulated) efficiency point. For example, some driving points at lower speeds have higher torque. In this case, these points could probably be regulated into the DDA to improve total efficiency.

The above considerations indicate that the future $\mathrm{HEV}$ transmission development trends might be as follows. First, as the regulation target is an interval rather than a point, more gears are not necessary for HEVs. Second, to regulate a wide speed range into a narrow speed interval, transmission ratios must be redesigned.

\subsection{Transmission Improvement}

Verification models were built to support evaluation of the two major directions of HEV transmission development. The SP HEV system could be considered as an electric CVT between the engine and wheels. This is because all the points outside the DDA can be regulated to the best efficiency point (2100 rpm/100 N m), just like a continuous speed change. In contrast, in the P2 HEV system, the transmission changes speed in steps, and speed intervals can be achieved only by regulating the efficiency points. From the perspective of cost, it is suggested that a step-changing transmission is preferable when applying the DDA method. Recall that the FC value of an SP HEV with transmission is $4.06 \mathrm{~L} / 100 \mathrm{~km}$, and that of a P2 HEV with transmission is $4.09 \mathrm{~L} / 100 \mathrm{~km}$. The slight change comes from the regulated points that are outside DDA. In the SP HEV system, these points are regulated to the best (40\%) efficiency point. In the P2 HEV system, the points are along the line nearest the best point, with a slightly lower average engine efficiency. Note that the P2 HEV system is constructed with fewer components, that is, engine, motor, and transmission. The SP HEV system with transmission has these components plus an additional generator. It should be noted that the commercial SP HEV cars of Honda have no transmission, as shown in Fig. 4. As the P2 HEV has a better cost performance, we further studied transmission applications based on P2 HEV systems.

A P2 HEV model with a three-speed transmission was built. The three-speed transmission was obtained by using the first, third, and fifth gears of the original five-speed transmission. The working point distribution is shown in Fig. 8 and compared with the five-speed transmission. The FC value is $4.09 \mathrm{~L} / 100 \mathrm{~km}$, which is equal to that of the five-speed version. In Fig. 8, it can be seen that the two models share similar point distributions because they share three gear sets. More points in the three-speed version are outside the DDA, and they are regulated along the best efficiency line. But the total number of these points is too small to influence the final FC result. Even for the points outside the DDA, the engine efficiency is still more than $30 \%$. Furthermore, the ratios of gears may not be optimal, and the gear shift strategy could 


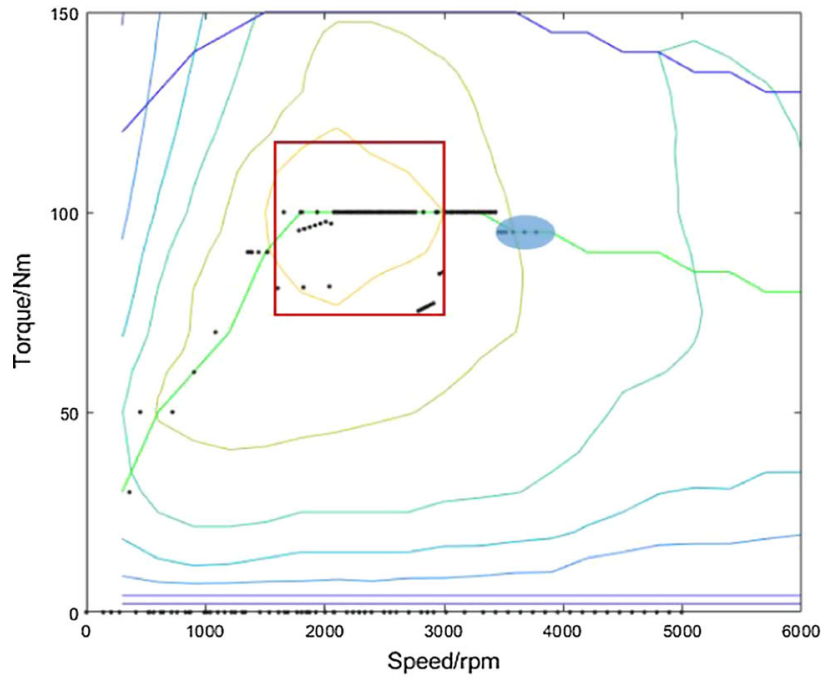

(a)

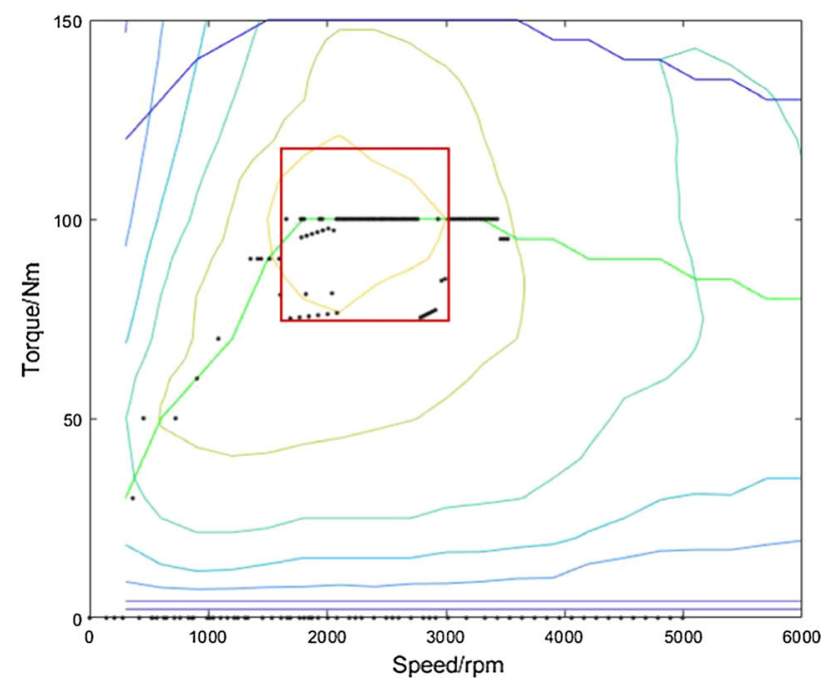

(b)

Fig. 8 Effect of reducing the number of gears in an HEV transmission. a Three-speed transmission driving points. b Five-speed transmission driving points

also be improved. Thus, the FC can still be further reduced. If the control strategy's potential is fully developed, the FC might meet even stricter $\mathrm{FC}$ regulations.

The results presented above support the utility of the HEV transmission development trends that we have proposed. With fewer gears but optimal ratios, the cost of HEV transmissions could be reduced and help increase HEV penetration in the vehicle market.

\section{Conclusions}

This paper described how the future function of HEV transmissions will be different, which will regulate engine speed into a certain speed interval, rather than to the best efficiency point for a given power. Development trends of HEV transmissions based on this new function were predicted, that is, that fewer gears will be used in HEV transmissions, but the gear ratios will be optimized. This conclusion is based on several HEV models, an energy management strategy considering total efficiency, and an engine working point distribution analysis. The presented DDA method made the engine drive the wheels directly in the high-efficiency area. In contrast, the rule-based strategies forced the engine to work along the best efficiency line in the efficiency map. The DDA method was verified by applying it to different HEV models. With fewer transmission gears, the total cost of an HEV will be reduced, which is beneficial for increasing market share.

Acknowledgements This study is sponsored by the National Natural Science Foundation of China (71403142, 71774100, 71690241), Young Elite Scientists Sponsorship Program of the Chinese Association for Science and Technology (YESS20160140), and Beijing Natural Science Foundation (9162008)

Open Access This article is distributed under the terms of the Creative Commons Attribution 4.0 International License (http://creativecomm ons.org/licenses/by/4.0/), which permits unrestricted use, distribution, and reproduction in any medium, provided you give appropriate credit to the original author(s) and the source, provide a link to the Creative Commons license, and indicate if changes were made.

\section{References}

1. Zhao, F., Su, R., Liu, Z.: Turning China into a stronger automotive country: an insight. China Machine Press, Beijing (2016)

2. Zhao, F., Su, R., Liu, Z.: Turning China into a stronger automotive country: an exploration. China Machine Press, Beijing (2017)

3. Zhao, F., Su, R., Liu, Z.: Turning China into a stronger automotive country: a practice. China Machine Press, Beijing (2017)

4. Liu, Z.: Fuquan Zhao's insights on automotive industry, vol. 1. China Machine Press, Beijing (2017)

5. Onat, N.C., Kucukvar, M., Tatari, O.: Conventional, hybrid, plugin hybrid or electric vehicles. State-based comparative carbon and energy footprint analysis in the United States. Appl. Energy 150, 36-49 (2015)

6. Shi, T., Zhao, F., Hao, H., et al.: Structure analysis and cost estimation of hybrid electric passenger vehicle and the application in china case. In: WCX World Congress Experience (2018)

7. Hawkins, T.R., Gausen, O.M., Strømman, A.H.: Environmental impacts of hybrid and electric vehicles-a review. Int. J. Life Cycle Assess. 17(8), 997-1014 (2012)

8. Atabani, A.E., Badruddin, I.A., Mekhilef, S., et al.: A review on global fuel economy standards, labels and technologies in the transportation sector. Renew. Sustain. Energy Rev. 15(9), 4586-4610 (2011)

9. Kaewunruen, S., Sussman, J.M., Matsumoto, A.: Grand challenges in transportation and transit systems. Front. Built Environ. 2, 4 (2016)

10. Zhou, M., Jin, H., Wang, W.: A review of vehicle fuel consumption models to evaluate eco-driving and eco-routing. Transp. Res. Part D Transp. Environ. 49, 203-218 (2016)

11. Gong, H., Wang, M.Q., Wang, H.: New energy vehicles in China: policies, demonstration, and progress. Mitig. Adapt. Strat. Glob. Change 18(2), 207-228 (2013) 
12. Zhao, F., Hao, H., Liu, Z.: Technology strategy to meet China's $5 \mathrm{~L} / 100 \mathrm{~km}$ fuel consumption target for passenger vehicles in 2020. Clean Technol. Environ. Policy 18(1), 7-15 (2016)

13. Liu, Z., Hao, H., Cheng, X., et al.: Critical issues of energy efficient and new energy vehicles development in China. Energy Policy 115, 92-97 (2018)

14. Hao, H., Wang, S., Liu, Z., et al.: The impact of stepped fuel economy targets on automaker's light-weighting strategy: The China case. Energy 94, 755-765 (2016)

15. Felgenhauer, M.F., Pellow, M.A., Benson, S.M., et al.: Evaluating co-benefits of battery and fuel cell vehicles in a community in California. Energy 114, 360-368 (2016)

16. Lo, K.: A critical review of China's rapidly developing renewable energy and energy efficiency policies. Renew. Sustain. Energy Rev. 29, 508-516 (2014)

17. Dimitrova, Z., Maréchal, F.: Environomic design of vehicle integrated energy system - application on a hybrid electric vehicle energy system. Chem. Eng. Trans. 39(475-480), 39 (2014)

18. Das, H.S., Tan, C.W., Yatim, A.H.M.: Fuel cell hybrid electric vehicles: a review on power conditioning units and topologies. Renew. Sustain. Energy Rev. 76, 268-291 (2017)

19. Raghavan, S.S., Khaligh, A.: Impact of plug-in hybrid electric vehicle charging on a distribution network in a Smart Grid environment. IEEE Pes Innovative Smart Grid Technologies. IEEE Comput. Soc. 1, 1-7 (2012)

20. Shi, D., Pisu, P., Chen, L., et al.: Control design and fuel economy investigation of power split HEV with energy regeneration of suspension. Appl. Energy 182, 576-589 (2016)

21. Lave, L.B., MacLean, H.L.: An environmental-economic evaluation of hybrid electric vehicles: Toyota's Prius versus its conventional internal combustion engine Corolla. Transp. Res. Part D Transp. Environ. 7(2), 155-162 (2002)

22. Nordelöf, A., Messagie, M., Tillman, A.M., et al.: Environmental impacts of hybrid, plug-in hybrid, and battery electric vehicles-What can we learn from life cycle assessment. Int. J. Life Cycle Assess. 19(11), 1866-1890 (2014)

23. Hannan, M.A., Azidin, F.A., Mohamed, A.: Hybrid electric vehicles and their challenges: a review. Renew. Sustain. Energy Rev. 29, 135-150 (2014)

24. Cummings, T., Thomas, H.B., Zachary, D.A.: The effect of trip preview prediction signal quality on hybrid vehicle fuel economy. IFAC-PapersOnLine 48(15), 271-276 (2015)

25. Morais, H., Sousa, T., Soares, J., et al.: Distributed energy resources management using plug-in hybrid electric vehicles as a fuelshifting demand response resource. Energy Convers. Manag. 97, 78-93 (2015)

26. Chen, F., Nathaniel, T., Nicole, K.: Electrification of roads: opportunities and challenges. Appl. Energy 150, 109-119 (2015)
27. Yuan, Z., Teng, L., Sun, F.: Comparative study of dynamic programming and Pontryagin's minimum principle on energy management for a parallel hybrid electric vehicle. Energies 6(4), 2305-2318 (2013)

28. Zhu, Y., Chen, Y., Tian, G.: A four-step method to design an energy management strategy for hybrid vehicles. In: Proceedings of the American Control Conference, IEEE, 1, 156-161 (2004)

29. Ghorbani, R., Bibeau, E., Zanetel, P.: Modeling and simulation of a series parallel hybrid electric vehicle using REVS. In: American Control Conference, IEEE, 1, 4413-4418 (2007)

30. Dongye, D., Deng, P.: Power-balancing instantaneous optimization energy management for a novel series-parallel hybrid electric bus. Chin. J. Mech. Eng. 25(6), 1161-1170 (2012)

31. Zhao, Z., Yu, Z., Yin, M., et al.: Torque distribution strategy for single driveshaft parallel hybrid electric vehicle. In: Intelligent Vehicles Symposium, IEEE, pp. 1350-1353 (2009)

32. Aghaei, J., Nezhad, A., Rabiee, A.: Contribution of plug-in hybrid electric vehicles in power system uncertainty management. Renew. Sustain. Energy Rev. 59, 450-458 (2016)

33. Kikuchi, J.: Stability modeling of HEV/EV electric drives as a small-scale distributed power system. In: Applied Power Electronics Conference and Exposition IEEE, 1, 2664-2671 (2015)

34. Wu, L., Wang, Y., Yuan, X.: Multiobjective optimization of HEV fuel economy and emissions using the self-adaptive differential evolution algorithm. IEEE Trans. Veh. Technol. 60(6), 2458-2470 (2011)

35. Zhang, P., Yan, F., Du, C.: A comprehensive analysis of energy management strategies for hybrid electric vehicles based on bibliometrics. Renew. Sustain. Energy Rev. 48, 88-104 (2015)

36. Montazeri-Gh, M., Mahmoodi-k, M.: Development a new power management strategy for power split hybrid electric vehicles. Transp. Res. Part D Transp. Environ. 37, 79-96 (2015)

37. Finesso, R., Ezio, S., Mattia, V.: Robust equivalent consumptionbased controllers for a dual-mode diesel parallel HEV. Energy Convers. Manag. 127, 124-139 (2016)

38. Li, L., Wang, X.Y., Xiong, R., et al.: AMT downshifting strategy design of HEV during regenerative braking process for energy conservation. Appl. Energy 183, 914-925 (2016)

39. Hannan, A., Azidin, A., Mohamed, A.: Light vehicle energy management system using multi-power sources. Prz. Elektrotech. 88(3), 197-204 (2012)

40. Hannan, A., Azidin, A., Mohamed, A.: Multi-sources model and control algorithm of an energy management system for light electric vehicles. Energy Convers. Manag. 62(5), 123-130 (2012) 